\title{
Objetivos de Desarrollo Sostenible y Elementos Cognitivos de Tesis de Posgrado, Amazonas, Perú, 2015 - 2019
}

\section{Sustainable Development Goals and Cognitive Elements of Graduate Thesis, Amazonas, Peru 2015 - 2019}

\author{
Rubén Walter Huaranga Soto ${ }^{1}$
}

\section{RESUMEN}

La pobreza, desigualdad y cambio climático son problemas que aquejan a la humanidad; que, a pesar del crecimiento económico, desarrollo tecnológico y alta producción de alimentos, no se han logrado resolver. Por ello, 193 países suscribieron los diecisiete Objetivos de Desarrollo Sostenible como parte de la Agenda 2030. Siendo las cosas así, resulta claro, deberían de conllevar al desarrollo social y económico para alcanzar el equilibrio entre la prosperidad y la protección del planeta a través de políticas implementadas por los estados firmantes. Sin embargo, han sido dejados de lado en la mayoría de las tesis a nivel de posgrado. En ese sentido, el presente trabajo pretende determinar en qué porcentaje dichos objetivos han sido abordados en los elementos cognitivos de las tesis de la Escuela de Posgrado de la Universidad Nacional Toribio Rodríguez de Mendoza; 2015-2019. Para ello se recopilaron la totalidad de tesis de posgrado y se identificaron los objetivos considerados en las tesis. Se aplicó una ficha de cotejo para contrastar la implementación del estudio y establecer la relación directa, indirecta o ninguna de los objetivos con los elementos cognitivos. Se advirtió que once de los diecisiete objetivos fueron considerados en los títulos de las tesis; sin embargo, solo un tercio de los Elementos Cognitivos de las Tesis estaban relacionadas en forma directa con los Objetivos de Desarrollo Sostenible y en contraste, otro tercio, no existe relación.

Palabras clave: Objetivos de desarrollo; desarrollo sostenible; tesis de posgrado.

\begin{abstract}
Poverty, inequality, and climate change are problems that afflict humanity; that despite economic growth, technological development, and high food production; they have not been able to solve. For this reason, 193 countries signed the seventeen Sustainable Development Goals as part of the 2030 Agenda. This being the case, it is clear, that they should lead to social and economic development to achieve a balance between prosperity and the protection of the planet through policies implemented by the signatory states. However, these have been neglected in most graduate-level theses. In this sense, the present work tries to determine in what percentage these objectives have been approached in the cognitive elements of the theses of the Graduate School of the Universidad Nacional Toribio Rodríguez de Mendoza, 2015-2019. For this, all the postgraduate theses were compiled, and the objectives considered in the theses were identified. A check sheet was applied to contrast the implementation of the study and establish the direct, indirect or none of the objectives with the cognitive elements. It was noted that eleven of the seventeen objectives were considered in the thesis titles; however, only a third of the Cognitive Elements of the Theses were directly related to the Sustainable Development Goals and in contrast, another third, there is no relationship.
\end{abstract}

Keywords: Cognitive; development, sustainable, thesis.

\footnotetext{
${ }^{1}$ Magister en Derecho Civil y Comercial y Magister en Gestión y Planeamiento Educativo de la Universidad Nacional Hermilio Valdizán de Huánuco (2004-2005. Docente de pregrado y posgrado de la Universidad Nacional Toribio Rodríguez de Mendoza de Amazonas, Perú. email:walter.huaranga@untrm.edu.pe.
} 


\section{INTRODUCCIÓN}

Las brechas de pobreza y extrema pobreza son los temas fundamentales que requieren atención. Aproximadamente contamos con unos 2,500 millones de personas en condición de pobreza; de los cuales unos 1,000 millones, en extrema pobreza, quienes buscan cada día la manera de sobrevivir. En tanto que hay unos 6,5 millones de niños que mueren antes de cumplir los cinco años por causa de enfermedades que son prevenibles o curables (Sachs, 2015, pp. 559-560). Mientras que, en el Informe de las Naciones Unidas del 2015 , establece que son 836 millones de niños; en el Informe de Desarrollo sostenible 2019, señala que en 2017 eran 821 millones que sufrían de desnutrición frente a los 784 millones en el 2015. Estos problemas se encuentran relacionados con otros como la alimentación, la desigualdad e inclusión social, cambio climático, acceso a la salud y educación, desigual desarrollo económico, la discriminación en sus diversas formas, conflictos bélicos, entre otros que afectan la paz y concordia que la humanidad necesita atender para una vida en paz y prosperidad.

En atención a la problemática mundial mencionada, la Agenda 2030 que contiene diecisiete Objetivos de Desarrollo Sostenible (ODS), tal como se puede apreciar en la Tabla 1, fue suscrita por 193 países el 2015, entre ellos nuestro país, comprometiéndose a trabajar en la erradicación de la pobreza y las desigualdades, la conservación y protección del planeta y el buen uso de los recursos naturales. La Agenda 2030 "es un plan de acción en favor de las personas, el planeta y la prosperidad. ... Los Objetivos y las metas son de carácter integrado e indivisible y conjugan las tres dimensiones del desarrollo sostenible: económica, social y ambiental" (Naciones Unidas, 2015, p.1). En tal sentido, el país asumió el compromiso de desarrollar las actividades de planificación en las entidades públicas y privadas basándose en los Objetivos de Desarrollo Sostenible. Razón por lo que las Escuelas de posgrado (EPG) deben realizar la gestión, formación, capacitación, especialización e investigación en relación con la Agenda 2030.
Tabla 1. Los Objetivos de Desarrollo Sostenible por bloques

\begin{tabular}{|c|c|}
\hline Bloques & Objetivos \\
\hline $\begin{array}{c}\mathbf{P} \\
\mathbf{E} \\
\mathbf{R} \\
\mathbf{S} \\
\mathbf{O} \\
\mathbf{N} \\
\mathbf{A} \\
\mathrm{S}\end{array}$ & $\begin{array}{l}\text { 1. Poner fin a la pobreza en todas sus formas y } \\
\text { en el mundo. } \\
\text { 2. Poner fin al hambre, lograr la seguridad } \\
\text { alimentaria y la mejora de la nutrición y } \\
\text { promover la agricultura sostenible. } \\
\text { 3. Garantizar una vida sana y promover el } \\
\text { bienestar de todos a todas las edades. } \\
\text { 4. Garantizar una educación inclusiva y } \\
\text { equitativa de calidad y promover oportunidades } \\
\text { de aprendizaje permanente para todos. } \\
\text { 5. Lograr la igualdad de género y empoderar a } \\
\text { todas las mujeres y las niñas }\end{array}$ \\
\hline $\begin{array}{l}\mathbf{P} \\
\mathbf{L} \\
\mathbf{A} \\
\mathbf{N} \\
\mathbf{E} \\
\mathbf{T} \\
\mathbf{A}\end{array}$ & $\begin{array}{l}\text { 6. Garantizar la disponibilidad y la gestión } \\
\text { sostenible del agua y el saneamiento para todos. } \\
\text { 12. Garantizar modalidades de consumo y } \\
\text { producción sostenible. } \\
\text { 13. Adoptar medidas urgentes para combatir el } \\
\text { cambio climático y sus efectos. } \\
\text { 14. Conservar y utilizar sosteniblemente los } \\
\text { océanos, los mares y los recursos marinos para el } \\
\text { desarrollo sostenible. } \\
\text { 15. Proteger, restablecer y promover el uso } \\
\text { sostenible de los ecosistemas terrestres, } \\
\text { gestionar sosteniblemente los bosques, luchar } \\
\text { contra la desertificación, detener e invertir la } \\
\text { degradación de las tierras y detener la pérdida de } \\
\text { biodiversidad. }\end{array}$ \\
\hline $\mathbf{P}$ & $\begin{array}{l}\text { 7. Garantizar el acceso a una energía asequible, } \\
\text { fiable, sostenible y moderna para todos. }\end{array}$ \\
\hline $\begin{array}{l}\mathbf{R} \\
\mathbf{O} \\
\mathrm{S}\end{array}$ & $\begin{array}{l}\text { 8. Promover el crecimiento económico } \\
\text { sostenido, inclusivo y sostenible, el empleo } \\
\text { pleno y productivo y el trabajo decente para }\end{array}$ \\
\hline $\begin{array}{l}\mathbf{P} \\
\mathbf{E} \\
\mathbf{R}\end{array}$ & $\begin{array}{l}\text { todos. } \\
\text { 9. Construir infraestructuras resilientes, } \\
\text { promover la industrialización inclusiva y } \\
\text { sostenible y fomentar la innovación. }\end{array}$ \\
\hline $\begin{array}{l}\text { I } \\
\text { D } \\
\text { A } \\
\text { D }\end{array}$ & $\begin{array}{l}\text { 10. Reducir la desigualdad en los paises y entre } \\
\text { ellos. } \\
\text { 11. Lograr que las ciudades y los asentamientos } \\
\text { humanos sean inclusivos, seguros, resilientes y } \\
\text { sostenibles. }\end{array}$ \\
\hline $\begin{array}{l}\mathbf{P} \\
\mathbf{A} \\
\mathbf{Z}\end{array}$ & $\begin{array}{l}\text { 16. Promover sociedades pacificas e inclusivas } \\
\text { para el desarrollo sostenible, facilitar el acceso a } \\
\text { la justicia para todos y construir a todos los } \\
\text { niveles instituciones eficaces e inclusivas que } \\
\text { rindan cuentas. }\end{array}$ \\
\hline $\begin{array}{l}\text { ASOCIA- } \\
\text { CIONES }\end{array}$ & $\begin{array}{l}\text { 17. Fortalecer los medios de implementación y } \\
\text { revitalizar la Alianza Mundial para el Desarrollo } \\
\text { Sostenible. }\end{array}$ \\
\hline
\end{tabular}

La educación superior universitaria debe considerar en sus aportes el desarrollo sostenible. En tal sentido, las tesis de investigación deberían de estar alineadas a la Agenda 2030. La formulación requiere que compatibilicen como parte básica de la metodología la implementación de la agenda.

Por tales razones, las investigaciones deben tomar en cuenta los diecisiete ODS. Estos objetivos deben estar presentes en una de las secciones de investigación donde se exponen conocimientos vigentes, referenciales, coincidentes o discrepantes, logrados o no y en las ideas sugerentes producto de la investigación. En la introducción, ya que tanto en el problema, en la importancia, así como en los antecedentes encontramos conocimiento relacionado con la realidad. En la discusión, ya que en ella contrastamos lo que existe como conocimiento frente a lo que se ha logrado visibilizar, cuya relación podría 
ser de coincidencias o discrepancias con otras perspectivas sobre el tema de investigación; donde también se pone en evidencia las consecuencias, así como las limitaciones de la investigación. En la conclusión, porque establece el arribo a nuevos conocimientos o demostraciones logradas, las limitaciones y el aporte al conocimiento. En la recomendación, dado que en ella se presenta nuevas posibilidades que no han sido atendidas en la investigación, ya sea dentro del campo de investigación o académico o político. En las referencias bibliográficas, ya que indican cuáles son las fuentes y sustento de la investigación, desde la planificación hasta la presentación del informe de tesis; además permite acercarnos al conocimiento existente, fuente de las bases teóricas o de contrastación de otras hipótesis. Todos estos espacios, a partir de este momento, se denominarán Elementos Cognitivos de la Tesis (ECT), tal como puede observarse en la Tabla 2, los cuales sirvieron para contrastar la relación con los ODS.

Para llegar a conocer si es cierto la afirmación de que las tesis se encuentran de espaldas a la problemática descrita; se describe y explica la relación entre los ECT y los ODS. Por ello la importancia de la investigación que espera que los ODS se encuentren comprendidos en el contenido de los ECT de las tesis de la Escuela de Posgrado de la UNTRM de Amazonas, 2015-2019.

Tabla 2. Estructura de características de los Elementos Cognitivos de las Tesis.

\begin{tabular}{|c|c|}
\hline Elementos & Características \\
\hline INTRODUCCIÓN & $\begin{array}{l}\text { Problema de investigación } \\
\text { Importancia } \\
\text { Antecedentes }\end{array}$ \\
\hline DISCUSIÓN & $\begin{array}{l}\text { Opinión sobre sus resultados } \\
\text { Coincidencias con otras } \\
\text { investigaciones } \\
\text { Discrepancia con otras } \\
\text { investigaciones } \\
\text { Consecuencias teóricas del trabajo } \\
\text { Exposición de limitaciones }\end{array}$ \\
\hline CONCLUSIONES & $\begin{array}{l}\text { Demostraciones logradas } \\
\text { Declaración de problemas no } \\
\text { resueltos } \\
\text { Presentación de limitaciones } \\
\text { Aportes a la comunidad científica }\end{array}$ \\
\hline $\begin{array}{l}\text { RECOMENDA- } \\
\text { CIONES }\end{array}$ & $\begin{array}{l}\text { Para el campo aplicado } \\
\text { Para el campo académico } \\
\text { Para el campo política }\end{array}$ \\
\hline $\begin{array}{l}\text { REFERENCIAS } \\
\text { BIBLIOGRÁFICAS }\end{array}$ & $\begin{array}{l}\text { Libros } \\
\text { Artículos } \\
\text { Tesis } \\
\text { Otros } \\
\end{array}$ \\
\hline
\end{tabular}

En la región no existen investigaciones relacionadas con el tema. Sin embargo, para atender dicho trabajo, se parte de la idea de que "Si sumamos los esfuerzos de todos, podemos convertirnos en la primera generación que ponga fin a la pobreza extrema y en la última que se enfrente al cambio climático como una amenaza existencial" (Sachs, 2015 , p.14). Dicha afirmación resalta la pobreza extrema y el cambio climático. Sin embargo, estos temas presentan paradojas, ya que hay países pobres que cuentan con riqueza de biodiversidad y de materias primas frente a países ricos que carecen de ellas. Países ricos que ostentan tecnología para explotar, procesar, distribuir y posibilitar el desarrollo equilibrado; en tanto los países pobres carecen de ello. Además, los países desarrollados generan mayor contaminación ambiental frente a los países pobres. No obstante, Sachs (2015) insistía: “... el aspecto normativo del proyecto de desarrollo sostenible se orienta hacia cuatro objetivos definitorios de una buena sociedad: la prosperidad económica; la inclusión y cohesión social; la sostenibilidad ambiental; y la buena gobernanza por parte de...los gobiernos y las empresas" (p. 21); además, nos dice: “... una vida digna combina el desarrollo económico, la inclusión social y la sostenibilidad ambiental" (Sachs, 2015, p. 15).

De igual importancia, sobre formas de acercamiento a los objetivos, encontramos una guía para la implementación que establece una propuesta para la integración de los ODS con la planificación nacional, la misma que consideran tres ejes: Educación; Salud; Desarrollo Económico. También se encuentra las cuatro prioridades que deben realizar la Comisión Económica para América Latina y el Caribe (CEPAL) a fin de implementar y realizar el seguimiento de la Agenda 2030; entre ellas, el potenciar el análisis de los medios de implementación de la Agenda 2030 a nivel regional; y apoyar la integración de los ODS en los planes nacionales de desarrollo y en los presupuestos (Naciones Unidas, 2018, p. 6).

En cuanto al rol de la universidad frente al desarrollo sostenible; es ineludible el compromiso de las universidades en constituirse en agentes de cambio para posibilitar el desarrollo sostenible, generando facilidades para crear conocimiento acompañado de la formación del talento humano, con ética y praxis de protección el ambiente, con actitud crítica en la construcción de un futuro sostenible para todos los habitantes que se encuentran con limitaciones para atender los temas de pobreza, inequidad e injusticia social, reducción de la biodiversidad y abandono de las tierras cultivables, los cinturones de miseria en las ciudades. (Valerio, 2012, pp. 10-11). Asimismo, sobre la universidad y los ODS, nos encontramos con que "La prospectiva del tema plantea una institución más comprometida con el entorno, con la sociedad, con el hombre y sus problemas. De esta convivencia se espera que el sistema e instituciones de educación 
superior, en un tránsito histórico, dé respuestas más asertivas desde la pertinencia social" (Henríquez, 2018, p. 118).

Lo que se ha observado es que los trabajos de investigación no toman en cuenta los ODS; ya que las instituciones tampoco los toman en cuenta ni los incorporan como políticas de gestión establecidas por el Estado. Las investigaciones no están orientadas a la generación de conocimientos para proponer alternativas de solución a la problemática de la sociedad tomando en consideración la Agenda 2030 .

En función de lo anteriormente vertido, el presente estudio busca describir la relación de las tesis de la EPG con los ODS; resultados que servirán como referente para la toma de decisiones en la implementación de los ODS en la EPG, así como para la gestión y desarrollo de la investigación en las EPG durante la presente década. Temática que no se ha tomado en cuenta como política de investigación. Además, se justifica porque posibilita elevar el nivel de investigación y compromiso con la problemática de la humanidad; igualmente posibilita realizar investigaciones de forma, multi metódica, interdisciplinaria, interinstitucional, transdisciplinaria y holística.

\section{MATERIAL Y MÉTODOS}

Se realizó una investigación descriptiva correlacional; para ello primero se elaboró un cuadro (Tabla 1) sobre la Agenda 2030 con los diecisiete ODS ordenados por bloques. Luego se estableció los elementos y características de los ECT (Tabla 2). El estudio se inició con la identificación de los títulos de tesis en relación con los ODS; luego, guiados por las ideas de qué nadie se quede atrás y desarrollo sostenible que guían y sirven de fundamento, se realizó el cotejo de los diecisiete ODS con los ECT.

Las tesis consultadas correspondieron a la EPG de la UNTRM, las cuales correspondieron a los programas de maestrías y doctorado mostradas en la Figura 1; donde se encontró que, a la fecha de finalización del trabajo, la universidad contaba con un doctorado y diecisiete maestrías en funcionamiento del total de 21 maestrías autorizadas. Estos reportaron 46 tesis de maestría sustentadas y aprobadas de ocho maestrías; las otras nueve no cuentan con ninguna tesis, así como tampoco en el doctorado.

Figura 1. Tesis de maestrías y doctorado en la Escuela de Posgrado

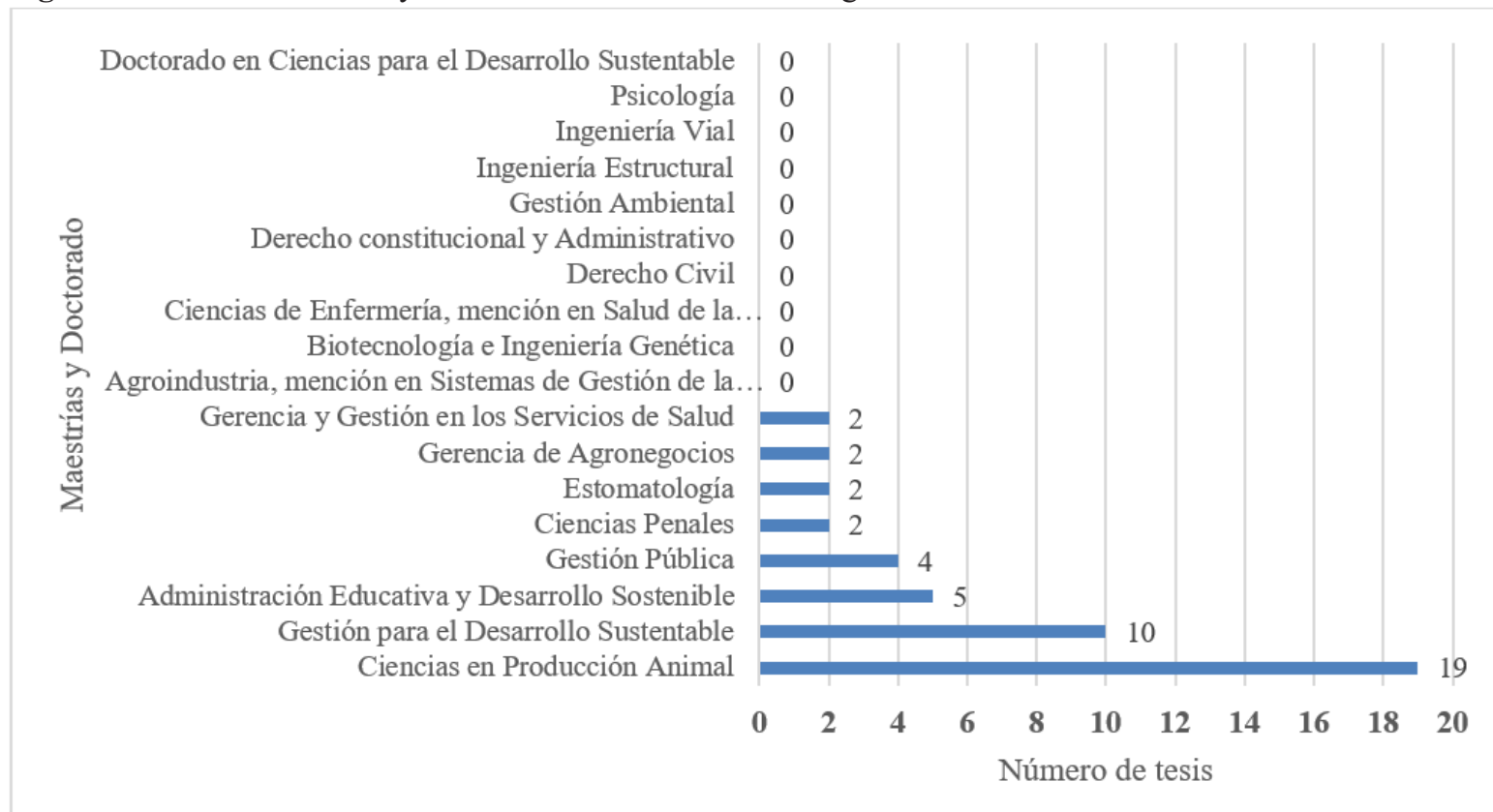

En ese sentido, la población y muestra son las 46 tesis sustentadas y registradas en el Libro de Registro de Grados de la Escuela de Posgrado y en el Repositorio de la UNTRM hasta fines del 2019.

El análisis de contenido de los trabajos recopilados, usamos dos fichas de cotejo de resultados de las relaciones entre variables observadas, los ODS y
ECT, de acuerdo con lo propuesto en el trabajo de Ander-Egg (1980, pp. 94-295). La primera contiene dos secciones; los títulos de las tesis por un lado y por otro los diecisiete objetivos agrupados en cinco bloques; lo que permitió obtener los datos de relación entre títulos y los objetivos. La segunda contiene dos secciones; la primera, los ECT con sus características 
y la segunda sección nos permitió extraer información de sobre la existencia de una relación directa, indirecta o ninguna con los ODS.

Los datos recopilados se analizaron mediante estadística descriptiva, agrupando los principales resultados a través de la presentación de tablas y figuras de la información obtenida en el proceso de investigación, que permitió establecer la existencia o no de las relaciones entre ODS y ECT de las 46 tesis de posgrado.

ECT, de acuerdo con lo propuesto en el trabajo de Ander-Egg (1980, pp. 94-295). La primera contiene dos secciones; los títulos de las tesis por un lado y por otro los diecisiete objetivos agrupados en cinco bloques; lo que permitió obtener los datos de relación entre títulos y los objetivos. La segunda contiene dos secciones; la primera, los ECT con sus características y la segunda sección nos permitió extraer información de sobre la existencia de una relación directa, indirecta o ninguna con los ODS.

Los datos recopilados se analizaron mediante estadística descriptiva, agrupando los principales resultados a través de la presentación de tablas y figuras de la información obtenida en el proceso de investigación, que permitió establecer la existencia o no de las relaciones entre ODS y ECT de las 46 tesis de posgrado.

\section{RESULTADOS}

En la siguiente tabla, se puede apreciar que el registro fue de cuarenta y seis tesis sustentadas en la Escuela de Posgrado de ocho maestrías (Tabla 3). La mayoría de las tesis sustentadas, total 19, pertenecían a la maestría de Ciencias en producción animal, seguida de 10 tesis en Gestión para el desarrollo sustentable.

Tabla 3. Distribución de las tesis por maestrías en la Escuela de Posgrado

\begin{tabular}{lcccccc}
\hline \multicolumn{1}{c}{ Maestrías } & \multicolumn{3}{c}{ Tesis } & \multicolumn{2}{c}{ Total } \\
\cline { 2 - 6 } & 2015 & 2016 & 2017 & 2018 & 2019 \\
\hline Administración educativa y desarrollo sostenible & 1 & 1 & 0 & 1 & 2 & 5 \\
Ciencias en producción animal & 0 & 1 & 0 & 17 & 1 & 19 \\
Ciencias penales & 0 & 0 & 0 & 2 & 0 & 2 \\
Estomatología & 0 & 0 & 0 & 2 & 0 & 2 \\
Gerencia de agronegocios & 0 & 0 & 1 & 1 & 0 & 2 \\
Gerencia y gestión en los servicios de salud & 0 & 0 & 1 & 1 & 0 & 2 \\
Gestión para el desarrollo sustentable & 0 & 1 & 1 & 6 & 2 & 10 \\
Gestión pública & 1 & 1 & 0 & 2 & 0 & 4 \\
\hline \multicolumn{1}{c}{ TOTAL } & 2 & 4 & 3 & 30 & 5 & $\mathbf{4 6}$
\end{tabular}

Según la información recopilada, sólo ocho maestrías de las diecisiete que se encuentran en funcionamiento en la EPG de la UNTRM cuentan con 46 tesis aprobadas; cuyos títulos se relacionan con once ODS; mientras que ningún título con seis de los siguientes ODS: Igualdad de género, Producción y consumo responsable, Vida submarina, Reducción de las desigualdades, Ciudades y comunidades sostenibles ni con Alianzas para lograr los objetivos.
De las cuarenta y seis tesis; once títulos tienen relación con el objetivo Industria, innovación e infraestructura; nueve, con Trabajo decente y crecimiento económico. Además, se observó que veintiuno títulos de tesis están relacionados con el bloque prosperidad; sin embargo, ni un solo título relacionado a la reducción de las desigualdades ni con ciudades y comunidades sostenibles. Apenas una tesis relacionada con energía asequible y no contaminante (Tabla 4).

\begin{tabular}{|c|c|c|c|c|c|}
\hline \multirow{2}{*}{ Bloques } & \multirow{2}{*}{ Objetivos } & \multicolumn{4}{|c|}{ Tesis } \\
\hline & & fi & $\%$ & $\mathrm{Fi}$ & $\%$ \\
\hline \multirow{5}{*}{ PERSONAS } & 1. Fin de la pobreza & 1 & 2 & \multirow{5}{*}{10} & \multirow{5}{*}{22} \\
\hline & 2. Hambre cero & 3 & 7 & & \\
\hline & 3. Salud y bienestar & 2 & 4 & & \\
\hline & 4. Educación de calidad & 4 & 9 & & \\
\hline & 5. Igualdad de género & 0 & 0 & & \\
\hline \multirow{5}{*}{ PLANETA } & 6. Agua limpia y saneamiento & 5 & 11 & \multirow{5}{*}{9} & \multirow{5}{*}{20} \\
\hline & 12. Producción y consumo responsables & 0 & 0 & & \\
\hline & 13. Acción por el clima & 3 & 7 & & \\
\hline & 14. Vida submarina & 0 & 0 & & \\
\hline & 15. Vida de ecosistemas terrestres & 1 & 2 & & \\
\hline \multirow{5}{*}{ PROSPERIDAD } & 7. Energía asequible y no contaminante & 1 & 2 & \multirow{5}{*}{21} & \multirow{5}{*}{46} \\
\hline & 8. Trabajo decente y crecimiento económico & 9 & 20 & & \\
\hline & 9. Industria, innovación e infraestructura & 11 & 24 & & \\
\hline & 10. Reducción de las desigualdades & 0 & 0 & & \\
\hline & 11. Ciudades y comunidades sostenibles & 0 & 0 & & \\
\hline PAZ & 16. Paz, justicia e instituciones sólidas & 6 & 13 & 6 & 13 \\
\hline \multirow[t]{2}{*}{ ASOCIACIONES } & 17. Alianzas para lograr los objetivos & 0 & 0 & 0 & 0 \\
\hline & TOTAL & 46 & 100 & 46 & 100 \\
\hline
\end{tabular}


De las cuarenta y seis tesis; los ODS fueron considerados en treinta y dos problemas y treinta y tres de importancia de la investigación de forma directa en el elemento cognitivo introducción; mientras que treinta y dos antecedentes, en forma indirecta. Así mismo; los objetivos fueron considerados en treinta y uno libros de forma indirecta en el elemento cognitivo referencia bibliográfica. En tanto que, cuarenta tesis no tienen relación de la declaración de problemas no resueltos con los ODS; de igual manera, treinta y nueve tampoco tienen relación o no se mencionan sobre la presentación de limitaciones en el elemento cognitivo conclusiones (Tabla 5).

Tabla 5. Relación de los elementos cognitivos de las tesis con los objetivos de desarrollo sostenible

\begin{tabular}{llrrr}
\hline & Elementos & \multicolumn{3}{c}{ Relación } \\
\cline { 3 - 5 } INTRODUCCION & Directa & Indirecta & Ninguna \\
& Problema de investigación. & 32 & 14 & 0 \\
& Importancia. & 33 & 13 & 0 \\
& Antecedentes. & 12 & 32 & 2 \\
\hline \multirow{2}{*}{ DISCUSION } & Opinión sobre sus resultados. & 22 & 14 & 10 \\
& Coincidencias con otras investigaciones. & 24 & 17 & 5 \\
& Discrepancia con otras investigaciones. & 20 & 8 & 18 \\
& Consecuencias teóricas del trabajo. & 12 & 13 & 21 \\
& Exposición de limitaciones. & 6 & 3 & 37 \\
\hline & Demostraciones logradas. & 26 & 15 & 5 \\
CONCLUSIONES & Declaración de problemas no resueltos. & 1 & 5 & 40 \\
& Presentación de limitaciones. & 5 & 2 & 39 \\
& Aportes a la comunidad científica. & 10 & 15 & 21 \\
\hline \multirow{2}{*}{ RECOMENDA- } & Para el campo aplicado. & 19 & 14 & 13 \\
CIONES & Para el campo académico. & 11 & 13 & 22 \\
& Para el campo político. & 12 & 17 & 17 \\
\hline \multirow{2}{*}{ REFERENCIA } & Libros. & 11 & 31 & 4 \\
BIBLIOGRAFICA & Artículos. & 16 & 27 & 3 \\
& Tesis. & 7 & 29 & 10 \\
\hline & Otros. & 11 & 28 & 7 \\
\hline & TOTAL & $\mathbf{2 9 0}$ & $\mathbf{3 1 0}$ & $\mathbf{2 7 4}$ \\
\hline
\end{tabular}

Los ODS en un treinta y tres por ciento de las tesis revisadas están comprendidos en forma directa en los ECT, un $35.5 \%$, en forma indirecta. Por el contrario, un $31.4 \%$ no se encuentran comprendidos o no se consideraron los objetivos en el contenido de los ECT (Figura 2).

Figura 2. Relación de los objetivos de desarrollo sostenible y los elementos cognitivos de las tesis.

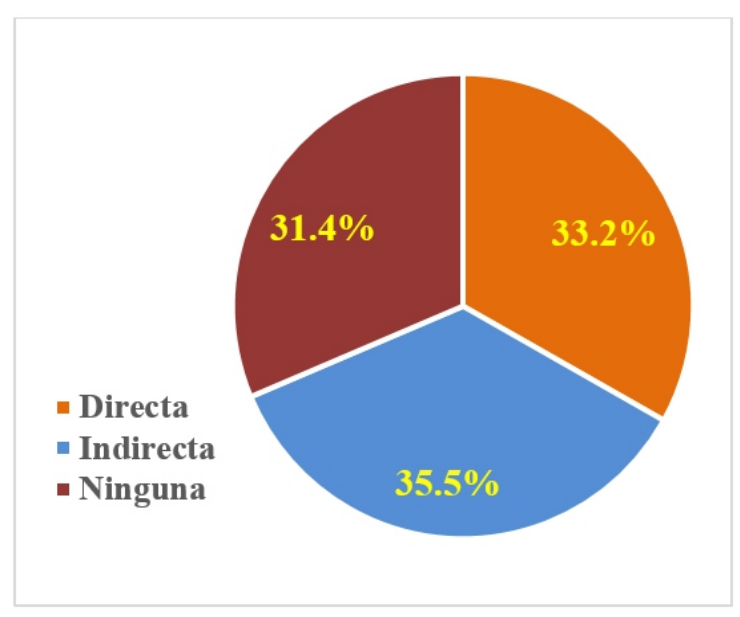

\section{DISCUSIÓN}

El presente trabajo no fue materia de anteriores estudios sobre el constructo elementos cognitivos de las tesis; en cambio sí hay trabajos sobre los objetivos de desarrollo sostenible relacionados con otros rubros. Además, es posible estudiar el tema con mayor amplitud y con la inclusión de otras variables. En este sentido, el objeto de investigación buscó describir y analizar la relación directa, indirecta o ninguna entre los ECT y los ODS de las tesis de posgrado de la Universidad Nacional Toribio Rodríguez de Mendoza. Nuestro estudio evidencia que, con los resultados descritos en el apartado precedente, las tesis guardan una muy baja relación con los objetivos de la Agenda 2030. En el último apartado, referido al tema central de nuestro trabajo, la relación de los ECT con los ODS es de gran valor en investigación, sin importar la entidad o investigador, ya que lo que se busca es atender a la problemática de la humanidad. Los resultados indican que aproximadamente un tercio $(33.2 \%)$ de los ECT están relacionados directamente con los ODS.

Cuando la investigación muestra estos resultados, se entiende que no se está cumpliendo o no se está tomando en cuenta las políticas públicas o no se realiza la debida socialización y sensibilización. Tal como Gómez (2018) considera que los ODS exponen respuestas al problema global de la desigualdad y la extrema pobreza, consumo no sostenible y la degradación ambiental; además, se constituye en una hoja de ruta para la articulación de políticas mundiales y que, entre los elementos críticos, para su aplicación, considera generar conocimiento científico. Además, enfatiza que si se quiere avanzar en la aplicación y cumplimiento de los ODS se deben tomar medidas ambiciosas y urgentes; decisiones claras y compromisos políticos precisos para que se produzcan avances significativos; así como medidas 
efectivas de transformación para mejorar el planeta y las condiciones de vida de sus habitantes.

En el apartado precedente; se ha realizado una estimación de la relación de los 46 títulos de las tesis con los objetivos de desarrollo sostenible como punto de partida del presente trabajo (Tabla 4); donde se destaca, como referente de investigación, el bloque prosperidad con veintiún títulos; once de los cuales relacionados con industria, innovación e infraestructura; nueve, a trabajo decente y crecimiento económico y solo un título, a energía asequible y no contaminante. Por el contrario, en relación con los otros objetivos; tenemos un título, fin de la pobreza; sin embargo, ningún título se refiere a igualdad de género, producción y consumo responsable, reducción de las desigualdades, ciudades y comunidades sostenibles ni con alianzas para lograr los objetivos. Desde nuestro punto de vista, el trabajo no desarrolló con profundidad este punto, ya que, al ser considerados los títulos como expresión de temas en forma genérica, podrían estar presentes relaciones con otros rubros, intereses o políticas de investigación; por lo tanto, queda pendiente la investigación de los títulos en relación con la justificación de la investigación.

Castillo y Pallavicini (2019), sostienen que las universidades son muy importantes en el desarrollo de la relación con la sociedad; tanto es así que la Universidad Nacional Autónoma de Nicaragua-Managua en cumplimiento con los ODS de la Agenda 2030, incorpora en todas sus acciones el bienestar y la creación de capacidades en las personas desde sus planes, estrategias y programas, para el permanente intercambio de saberes y conocimientos buscando la solución a las problemáticas de la sociedad desde la investigación, la internacionalización, la extensión y la gestión. No hay coincidencia con la investigación, ya que el trabajo presenta algunas debilidades como el hecho que no existe una equidad de producción de tesis por maestría; ocho de diecisiete maestrías cuentan con producción de tesis; diecinueve tesis son en producción animal y diez de gestión para el desarrollo sustentable.

Tal diferencia, con el mayor número de tesis, es porque la primera maestría se desarrolló con financiamiento de un programa de becas a tiempo completo. Además del análisis y contraste de la relación entre las variables, se encontró un título de tesis relacionado con el objetivo Energía Asequible y no contaminante; ningún título consideró reducción de las desigualdades ni ciudades y comunidades sostenibles del bloque prosperidad; tampoco sobre desigualdad ni la búsqueda de sostenibilidad y apenas un trabajo sobre energía no contaminante.

Cano-Gines (2018), Define a la Agenda 2030 como un proceso participativo multinivel y multiactor y en cuanto al rol de la universidad frente a los ODS, ésta se constituye en un actor importante en la Cooperación al Desarrollo y, asimismo, contribuye a la relación con otros agentes de cooperación para fomentar el trabajo en red. En tal sentido, la cooperación universitaria al desarrollo de la investigación, la innovación y gestión es necesaria. Afirma que, los ODS incluyen todas las ramas del conocimiento y debemos ser capaces de incidir en ello; de tal modo que, la generación de conocimientos en la universidad y su difusión se ponen al servicio de las acciones necesarias para producir cambios en beneficio de la toda la sociedad. Por ello la estrategia para un desarrollo sostenible ha de ser parte principal en la hoja de ruta de las universidades; la política universitaria debe tener como fin último conseguir una sociedad inclusiva y responsable que transforme el mundo. En esta parte, ninguna tesis consideró el bloque Asociaciones que tiene que ver con el ODS 17 Alianzas para lograr los objetivos.

Nuestro trabajo presenta como fortalezas que las tesis, como población de estudio, corresponden al periodo de cinco años, paralelos a la suscripción de la Agenda 2030; sin embargo, una de las debilidades es el haber incluido a la referencia bibliográfica, ya que en la observación solo se limitó a evaluar los títulos; consideramos que es insuficiente para arribar a conclusiones objetivas en este elemento; debería de realizarse una investigación sobre el contenido de cada texto o libro para comprobar la posibilidad de relación de los objetivos de desarrollo sostenible.

\section{CONCLUSIONES}

Basados en nuestro estudio, el análisis nos muestra que, existe una deficiente relación entre ODS y ECT en las tesis de posgrado de la UNTRM de Amazonas. Asimismo, se observan que ningún título de investigación consideró la reducción de las desigualdades ni ciudades y comunidades sostenibles del bloque prosperidad; ni temas de desigualdad ni la búsqueda de sostenibilidad; un título sobre energía no contaminante y ninguno consideró el bloque Asociaciones que tiene que ver con el Objetivo 17 Alianzas para lograr los objetivos. Por tanto, al encontrar sólo un tercio aproximadamente (33.2\%) de relación entre las variables, se puede afirmar que la relación es baja.

Como aporte o propuesta se estableció y describió a los Elementos Cognitivos de las Tesis; como espacios de la tesis donde se exponen los conocimientos vigentes, referenciales, coincidentes o discrepantes, logrados o no en la investigación y las ideas sugerentes producto de la investigación. No obstante, una limitación del trabajo se encuentra en que no se consideró la política de gestión ni de investigación de la EPG en relación con los ODS como política de gestión y desarrollo de la investigación que es importante para la presente década a fin de liderar la investigación en forma interdisciplinaria, multi metódica, interinstitucional, transdisciplinaria y holística; basada en la problemática de la humanidad.Por lo que se recomienda el desarrollo de 
investigaciones que permitan la incorporación de los objetivos de la Agenda 2030 como fuente y base de las investigaciones el presente estudio y hacer extensivo en beneficio de las entidades investigadoras académicas públicas o privadas.

\section{REFERENCIAS BIBLIOGRÁFICAS}

Ander-Egg, E. (1980). Técnicas de Investigación social. El Cid Editor.

Cano-Gines, A. (2018). El papel de la Universidad en la Cooperación Internacional al Desarrollo. Los ODS y el horizonte 2030. XVI Congreso Nacional Educación Comparada Tenerife. Recuperado el 25 de j u l i o d e 2020 . http://doi.org/10.25145/c.educomp.2018.1 6.082

Castillo, E. y Pallavicini, M. (2019). Avances de los Objetivos de Desarrollo Sostenible (ODS) en las Universidades: Caso UNANManagua. Revista Compromiso Social, 1(2), 25-34. Recuperado el 25 de julio de $\begin{array}{llll}2 & 0 & 2 & 0\end{array}$. nt_sostenibilidad.2019.v1.i1.1202

Gómez, C. (2018). Objetivos de Desarrollo Sostenible (ODS): una revisión crítica. Papeles de relaciones ecosociales y cambio global, (140), 107-118. Recuperado el 11 de e n e r o $\quad \mathrm{d} \mathrm{e} \quad 2020$. https://www.fuhem.es/media/cdv/file/bibli oteca/revista_papeles/140/ODS-revisioncritica-C.Gomez.pdf

Henríquez, P. (2018). El papel estratégico de la educación superior en el desarrollo sostenible de América Latina y el Caribe. Conferencia Regional de Educación superior de América Latina y el Caribe. Córdoba, 2018. Resúmenes ejecutivos. UNESCO-IESALO y UNO

Naciones Unidas. (2018). Guía metodológica: planificación para la implementación de la Agenda 2030 en América Latina y el Caribe. Comisión Económica para América Latina y el Caribe (CEPAL). Recuperado el 11 de enero de 2020. http://www.aecid.es/CentroDocumentacion/Documentos/Divulgaci\% C3\%B3n/Comunicaci\%C3\%B3n/S180055 6_es\%20(1).pdf

Naciones Unidas (2015). Objetivos del Desarrollo del Milenio. Informe 2015. Nueva York. Recuperado el 11 de enero de 2020. https://www.un.org/es/sections/general/do cuments/index.html

Naciones Unidas. (2015). La resolución 70/1 de la Asamblea General "Transformar nuestro mundo: la Agenda 2030 para el Desarrollo sostenible", A/RES/70/1 (21 de octubre de 2015). Recuperado el 11 de enero de 2020. https://undocs.org/es/A/RES/70/1

Sachs, J. (2015). La era del desarrollo sostenible. Ediciones DEUSTO.

Valerio, C. (2012). Las universidades latinoamericanas y caribeñas ante el reto del desarrollo sostenible. Revista Congreso Universidad. Vol. I, No. 1, 2012. Editorial Universitaria FÉLIX VALERA. Recuperado el 11 de enero de 2020. http://www.congresouniversidad.cu/revista /index.php/rcu/article/view/885/823 